\title{
Refractive changes in diabetic patients during intensive glycaemic control
}

\author{
Fumiki Okamoto, Hirohito Sone, Tomohito Nonoyama, Sachiko Hommura
}

\begin{abstract}
Aims-To evaluate the clinical course and the characteristics of transient refractive error occurring during intensive glycaemic control of severe hyperglycaemia.

Methods-28 eyes of patients with persistent diabetes were included in this prospective study. During the observation period, patients underwent general ophthalmological examination and A-mode scan ultrasonography was performed at each examination-at days 1,3 , and 7 , and then once every week or every other week until recovery of hyperopia.
\end{abstract}

Results-A transient hyperopic change occurred in all patients receiving improved control after hyperglycaemia. Hyperopic change developed a mean of 3.4 (SD 2.0) days after the onset of treatment, and reached a peak at 10.3 (6.1) days, where the maximum hyperopic change in an eye was $1.47(0.87) \mathrm{D}$ (range $0.50-3.75$ D). Recovery of the previous refraction occurred between 14 and 84 days after the initial assessment. There was a positive correlation between the magnitude of the maximum hyperopic change and (1) the plasma glucose concentration on admission $(p<0.01),(2)$ the $\mathbf{H b A}_{1 \mathrm{c}}$ level on admission $(p<0.005)$, (3) the daily rate of plasma glucose reduction over the first 7 days of treatment $(p<0.001)$, (4) the number of days required for hyperopia to reach its peak $(p<0.001)$, and (5) the number of days required for the development and resolution of hyperopic changes $(p<0.0001)$. There was a negative correlation between the maximum hyperopic change of an eye and baseline value of refraction $(p<0.01)$. During transient hyperopia, no significant changes were observed in the radius of the anterior corneal curvature, axial length, lens thickness, or depth of anterior chamber.

Conclusions-The degree of transient hyperopia associated with rapid correction of hyperglycaemia is highly dependent on the rate of reduction of the plasma glucose level. A reduction of refractive index in intraocular tissues, especially in lens, appears to be responsible for this hyperopic change.

(BrF Ophthalmol 2000;84:1097-1102)

Patients with diabetes mellitus (DM) can develop various ocular complications including cataract, optic neuropathy, uveitis, and keratopathy as well as retinopathy. In addition, transient refractive error occurs during the course of DM and is associated with treatment induced changes in the plasma glucose concentration. $^{1-8}$ Regarding the effect of chronic changes in plasma glucose, myopia associated with hyperglycaemia and hyperopia with hypoglycaemia have been reported to develop in diabetic patients. ${ }^{9}$ Some investigators have suggested that acute changes in plasma glucose for 1-2 months will cause hyperopia, ${ }^{13-6}$ while others have suggested that either myopia or hyperopia will occur when the plasma glucose level increases or decreases. ${ }^{2} 78$ Thus, the biological basis of refractive changes in the eyes of diabetic patients has not yet been established and the underlying mechanism is still unknown. While most published reports have been retrospective studies of a limited number of patients, the authors conducted a prospective study of 28 eyes of 14 diabetic patients who were hospitalised for severe hyperglycaemia and underwent glycaemic control, in an attempt to make an objective evaluation of refractive changes during treatment.

Patients, materials, and methods

The study was conducted on consecutive 28 eyes of 14 patients with DM who were admitted to Tsuchiura Kyodo Hospital between January 1998 and August 1998, and who had a plasma glucose level of $400 \mathrm{mg} / \mathrm{dl}$ or higher, or a $\mathrm{HbA}_{1 \mathrm{c}}$ of $12.0 \%$ or higher on admission. The subjects consisted of 11 men and three women, with a mean age of 51.2 (SD 13.1) years. Five of the 14 patients complained some symptoms including thirst, polydipsia and polyuria at the first visit to outpatient clinic, were diagnosed with diabetes mellitus, and admitted on that day. In four patients elevated plasma glucose levels were detected during health screening examination. The remaining five patients had been diagnosed as having DM 2-5 years before, but left the disease untreated after diagnosis. Excluded from this study were the patients who did not come to the hospital within 4 days after plasma glucose measurement on the initial examination at admission, and those who failed to undergo ophthalmological examinations according to the protocol because of poor general health.

The mean plasma glucose concentration at admission was 532 (SD 141) $\mathrm{mg} / \mathrm{dl}$ (range $286-824 \mathrm{mg} / \mathrm{dl}$ ) and the mean $\mathrm{HbA}_{1 \mathrm{c}}$ value was $11.9 \%(2.8 \%)$ (range $6.8-16.0 \%$ ). By the type of disease, 12 patients had non-insulin dependent diabetes mellitus (NIDDM) and two patients had insulin dependent diabetes mellitus (IDDM). Table 1 summarises the characteristics of the patients enrolled in this study.

An ophthalmological examination was conducted on admission (day 0 ), on days 1 , 3 , and 
Table 1 Clinical characteristics of the diabetic patients

\begin{tabular}{|c|c|c|c|c|c|c|c|c|c|}
\hline Patient No & $\begin{array}{l}\text { Sex } \\
(M / F)\end{array}$ & $\begin{array}{l}\text { Age } \\
\text { (years) }\end{array}$ & $\begin{array}{l}\text { Duration of } \\
\text { DM (years) }\end{array}$ & $\begin{array}{l}\text { Plasma glucose at } \\
\text { admission ( } \mathrm{mg} / \mathrm{dl})\end{array}$ & $H b A_{1 c}(\%)$ & $\begin{array}{l}\text { Type of } \\
D M\end{array}$ & Therapy & $\begin{array}{l}\text { Retinopathy } \\
(R / L)\end{array}$ & General complications \\
\hline 1 & M & 25 & ND & 286 & 14.2 & I & Insulin & $-1-$ & cholecystic adenomyosis \\
\hline 2 & M & 52 & 3 & 510 & 10.1 & II & Diet & $-1-$ & vasospastic angina \\
\hline 3 & $\mathrm{M}$ & 36 & 5 & 586 & 8.2 & II & Insulin & $-1-$ & chronic pancreatitis, alcoholic hepatitis \\
\hline 4 & $\mathrm{~F}$ & 72 & ND & 709 & 14.0 & II & Insulin & $-1-$ & hypertension \\
\hline 5 & M & 63 & ND & 542 & 12.9 & II & SU & $-1-$ & hypertension \\
\hline 6 & $\mathrm{M}$ & 32 & 2 & 545 & 12.4 & $\mathrm{I}$ & Insulin & $-1-$ & hyperlipidaemia \\
\hline 7 & M & 58 & ND & 508 & 13.5 & II & SU & $-1-$ & hepatitis \\
\hline 8 & M & 69 & ND & 824 & 16.0 & II & Insulin & $-1-$ & hypertension, hepatitis \\
\hline 9 & M & 49 & 5 & 730 & 15.6 & II & Insulin & $-1-$ & - \\
\hline 10 & $\mathrm{M}$ & 47 & ND & 470 & 12.4 & II & SU & $-1-$ & - \\
\hline 11 & $\mathrm{~F}$ & 49 & $\mathrm{ND}$ & 406 & 10.6 & II & Diet & $-1-$ & hypertension \\
\hline 12 & M & 56 & ND & 507 & 8.0 & II & SU & $-1-$ & alcoholic hepatitis \\
\hline 13 & $\mathrm{~F}$ & 51 & 3 & 419 & 12.0 & II & SU & NPDR/NPDR & hypertension, nephrosis \\
\hline & M & 58 & ND & 409 & 6.8 & II & Diet & $-1-$ & Wernicke-Korsakoff syndrome \\
\hline Mean (SD) & & $51(13)$ & & $532(141)$ & $11.9(2.8)$ & & & & \\
\hline
\end{tabular}

7, and then once every week or once every other week until recovery of hyperopia. The basic ophthalmological examination included assessment of visual acuity and intraocular pressure, slit lamp microscopic examination, funduscopy after mydriatics with $0.5 \%$ tropicamide, $0.5 \%$ phenylephrine hydrochloride, and $1 \%$ cyclopentolate hydrochloride. In addition, we measured the refraction and the radius of the anterior corneal curvature in all subject. In the eyes with astigmatism, spherical equivalent values were used as the refractive values. A-mode ultrasonography was performed by the same examiner seven times in each eye at each examination to measure the lens thickness, depth of the anterior chamber, vitreous length, and axial length. The mean value of the seven measurements was used for analysis of each biometric parameter. In addition, fluorescein fundus angiography was performed in the patients who had a change of the refractive error by 2 dioptres or more during the study.

The onset time of a change in refraction was defined as the day on which there was a change in the refractive change of 0.5 dioptre or more compared with the value on admission. If there was no further refractive change of 0.5 dioptre or more in the subsequent 2 weeks, determination was discontinued with the measurement used as the baseline value. This study was conducted in accordance with the Declaration of Helsinki, and informed consent was obtained from each subject.

To investigate the relation between decreasing plasma glucose concentration and changes in ocular variables, the daily rate of reduction of plasma glucose concentration over the first 3 days (V3) and over the first 7 days (V7) of treatment was calculated by the following equations:

$\mathrm{V} 3(\mathrm{mg} / \mathrm{d} 1 /$ day $)=($ pretreatment plasma glucose concentration - plasma glucose concentration on the 3rd day of treatment) $/ 3$, and

$\mathrm{V} 7(\mathrm{mg} / \mathrm{dl} /$ day $)=($ pretreatment plasma glucose concentration - plasma glucose concentration on the 7 th day of treatment) $/ 7$, respectively.

On the day of admission, patients were started on a diet of $25 \mathrm{kcal} / \mathrm{kg} /$ day, and were treated with diet alone, diet and insulin, or diet and sulfonylurea for glycaemic control during the admission. The plasma glucose level was determined six times a day before and after each meal nearly every day that the patient was hospitalised (mean duration of hospitalisation, 22 (10) days). In the above formulas for calculating the rate of reduction of plasma glucose concentration, the plasma glucose level with the highest value of the six measurements on the third and seventh days of treatment was used.

The results are presented as mean (SE), except where described otherwise. Statistical analyses of data were performed using paired Student's $t$ test, simple regression analysis, and stepwise multiple regression analysis. A p value of less than 0.05 was considered to be statistically significant.

\section{CASE REPORT (CASE 4)}

A 72 year old woman (height $149 \mathrm{~cm}$, body weight $79.5 \mathrm{~kg}, \mathrm{BMI} 35.8$ ) was seen at the outpatient clinic of the hospital with thirst and polyuria, and was admitted immediately for marked hyperglycaemia (plasma glucose 709 $\mathrm{mg} / \mathrm{dl}, \mathrm{HbA}_{1 \mathrm{c}} 14.0 \%$ ). The patient was found to have diabetic nephropathy, but did not have retinopathy or neuropathy. Following admission, sliding scale insulin therapy and a diet therapy of $1000 \mathrm{kcal} /$ day were instituted. On admission, her corrected visual acuity was 1.0 in both eyes. The refraction of her eyes was $-3.25 \mathrm{D}$ in the right eye and $-8.0 \mathrm{D}$ in the left eye. Hyperopic change appeared 7 days after the day of admission and the degree of

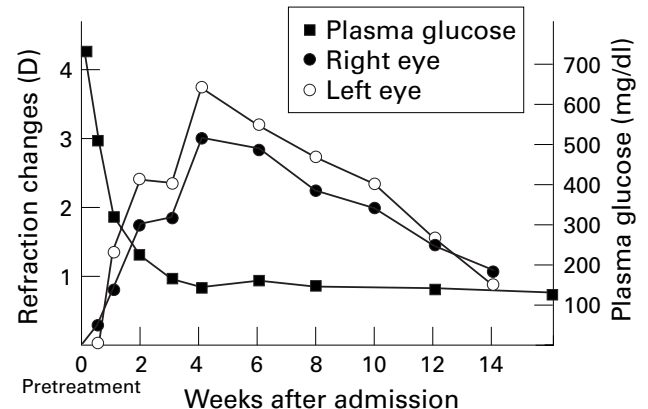

Figure 1 Change of refraction and of plasma glucose. concentration in a diabetic patient (case 4) over a period of 16 weeks after starting intensive glycaemic control on day 0 . The change of refraction in an eye at a given time point represents the difference between the refractive error on that day and the refractive error at the time of admission.

Transient bilateral hyperopic change was similar in both eyes, and appeared in response to a rapid reduction in plasma glucose. 
Table 2 Clinical course of transient hyperopic change

\begin{tabular}{llllll}
\hline $\begin{array}{l}\text { Patient } \\
\text { No }\end{array}$ & $\begin{array}{l}\text { Onset time of hyperopic } \\
\text { change (days after } \\
\text { admission) }\end{array}$ & $\begin{array}{l}\text { Peak time of hyperopic } \\
\text { change (days after } \\
\text { admission) }\end{array}$ & $\begin{array}{l}\text { Recovery time of } \\
\text { hyperopic change } \\
\text { after admission) }\end{array}$ & $\begin{array}{l}\text { Baseline refraction } \\
(\text { R/L; D) }\end{array}$ & $\begin{array}{l}\text { Maximum hyperopic } \\
\text { change }(R / L ; D)\end{array}$ \\
\hline 1 & 3 & 7 & 28 & $-1.6 /-1.9$ & $1.7 / 1.9$ \\
2 & 3 & 7 & 21 & $+0.5 /+0.4$ & $0.6 / 0.6$ \\
3 & 1 & 4 & 14 & $-3.5 /-3.6$ & $1.3 / 1.4$ \\
4 & 7 & 28 & 84 & $-3.3 /-8.0$ & $3.0 / 3.8$ \\
5 & 7 & 14 & 56 & $-1.0 /-0.1$ & $1.3 / 1.2$ \\
6 & 4 & 7 & 84 & $+0.3 / 0.0$ & $2.4 / 2.4$ \\
7 & 1 & 7 & 35 & $+0.1 /+0.3$ & $1.2 / 1.1$ \\
8 & 4 & 14 & 70 & $-0.3 /-0.3$ & $2.3 / 3.0$ \\
9 & 4 & 14 & $-0.3 /-0.7$ & $1.0 / 1.3$ \\
10 & 4 & 14 & 84 & $-1.0 /-1.0$ & $1.9 / 2.1$ \\
11 & 4 & 7 & 28 & $-5.0 /-0.3$ & $1.3 / 0.7$ \\
12 & 1 & 7 & 21 & $+0.3 /+0.5$ & $0.8 / 0.6$ \\
13 & 4 & 7 & 42 & $-0.9 /-1.4$ & $0.8 / 0.5$ \\
14 & 1 & 7 & 14 & $-1.1 /-0.3$ & $0.6 / 0.5$ \\
\hline
\end{tabular}

hyperopic change increased gradually there after. She complained of blurred vision with her glasses 2 weeks after admission. Maximal hyperopic change was seen at the fourth week, and showed a refraction of $-0.25 \mathrm{D}(\mathrm{RE})$ and $-4.25 \mathrm{D}$ (LE). The patient was discharged 1 month after admission, at which time the plasma glucose level had returned to the normal range. However, it took approximately 14 weeks for her refraction error to return to the baseline value on admission (Fig 1).
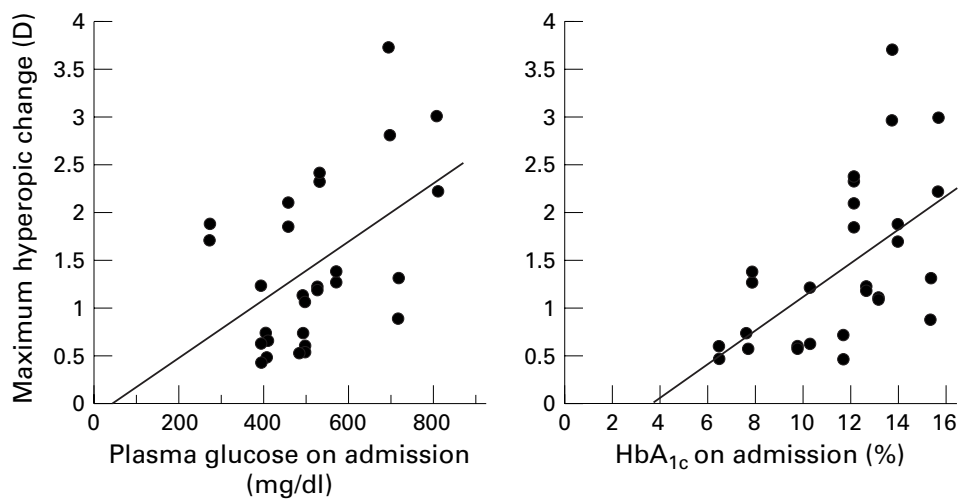

Figure 2 Relation between maximum hyperopic change of an eye (dioptres) and the plasma glucose concentration ( $\mathrm{mg} / \mathrm{dl}$ ) on admission (left). There was a significant positive correlation between them $(r=0.49, p<0.01)$. Relation between maximum hyperopic change of an eye (dioptres) and $\mathrm{Hb}_{1 c}(\%)$ on admission (right). There was a significant positive correlation between them $(r=0.57, p<0.005)$.
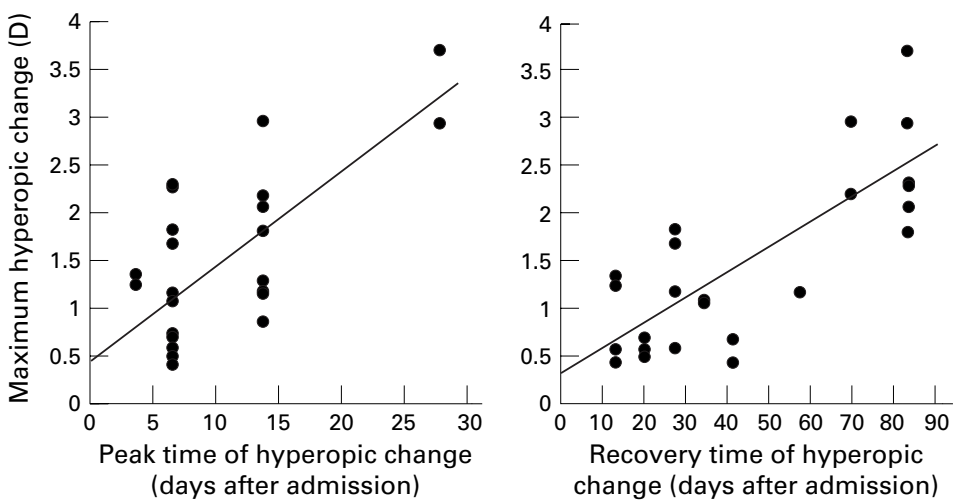

Figure 3 Relation between maximum hyperopic change of an eye (dioptres) and the day on which the maximum hyperopic change was attained (left). There was a significant positive correlation between them $(r=0.68, p<0.001)$. Relation between maximum hyperopic change of an eye (dioptres) and the day on which the patient recovered from hyperopia (right). There was a significant positive correlation between them ( $r=0.78$, $p<0.0001$ ).

\section{Results}

A transient hyperopic change occurred in all patients receiving improved control after hyperglycaemia. Hyperopic change appeared a mean of 3.4 (SD 2.0) days (range 1-7 days) after treatment was started; the maximum hyperopic change occurred 10.3 (6.1) days (range 4-28 days) after treatment was started. Refraction gradually returned to the baseline value over 44.7 (26.9) days (range 14-84 days) from the start of treatment. Treatment of hyperglycaemia produced a mean maximum hyperopic change in the 28 eyes of $1.47(0.87)$ dioptres. The largest magnitude of the refractive change was 3.75 dioptres in patient 4 (Table 2).

There was a significant positive correlation between maximum hyperopic change and plasma glucose concentration on admission $(r=0.49, \mathrm{p}<0.01$, Fig 2$)$. There was also a significant positive correlation between maximum hyperopic change and $\mathrm{HbA}_{1 \mathrm{c}}$ on admission $(r=0.57, \mathrm{p}<0.005$, Fig 2$)$.

There was a high correlation between maximum hyperopic change and the number of days it took for the eye to reach the maximum hyperopic change. An eye with a larger maximum change in refraction required a greater number of days to reach the maximum hyperopic change $(r=0.68, \mathrm{p}<0.001$, Fig 3$)$, and also a greater number of days from day of admission to recovery from hyperopic change to the baseline value of refraction $(r=0.78$, $\mathrm{p}<0.0001$, Fig 3).

A positive correlation was observed between the maximum hyperopic change and the daily rate of plasma glucose reduction over the first 7 days of treatment $(r=0.59, \mathrm{p}<0.001$, Fig 4$)$. However, there was no significant correlation between the maximum hyperopic change of an eye and daily rate of reduction over the first 3 days of treatment.

There was a negative correlation between the maximum hyperopic change and the stabilised refraction after treatment $(r=-0.48$, $\mathrm{p}<0.01$, Fig 5). This indicates that the greater the degree of myopia of a patient, the larger the hyperopic change that would occur during glycaemic control in diabetic patients.

The radius of the anterior corneal curvature, axial length, anterior chamber depth, and lens thickness at the peak time of hyperopic change 


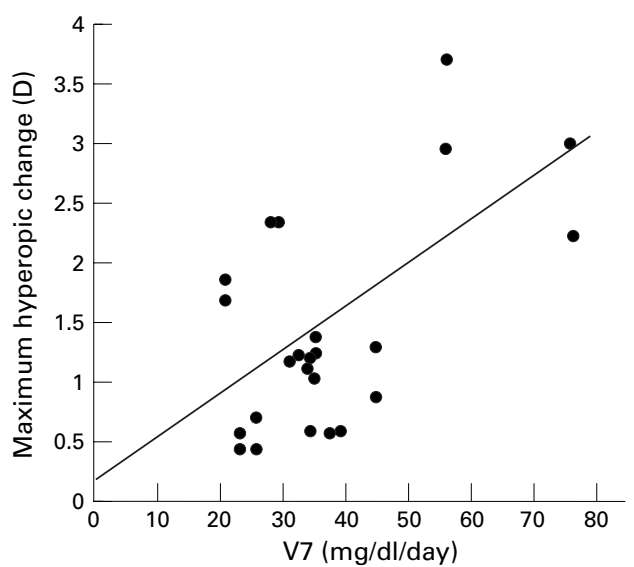

Figure 4 Relation between maximum hyperopic change of an eye and the daily rate of plasma glucose reduction over the first 7 days of treatment. There is a positive correlation between them $(r=0.59, p<0.001)$.

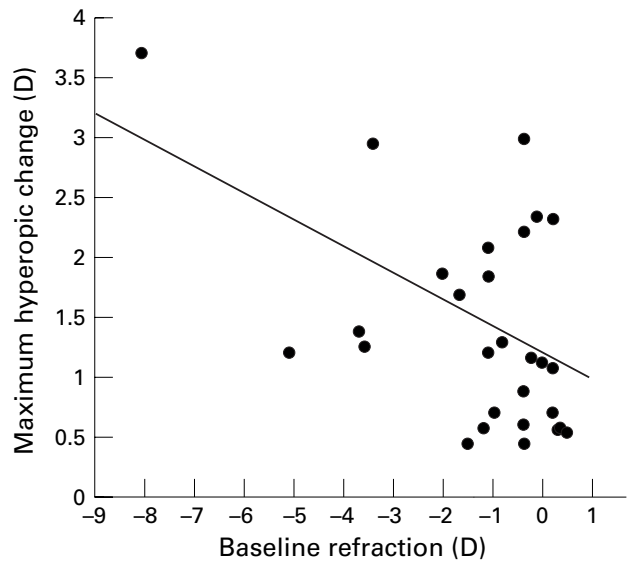

Figure 5 Relation between maximum hyperopic change of an eye and baseline refraction of the eye. There is a negative correlation between them $(r=-0.48, p<0.01)$.

did not differ from the respective value measured before treatment was started (Table 3).

Fluorescein fundus angiography was performed when the hyperopic change had reached 2 dioptres or more, and again after the refraction had stabilised. Fundus abnormalities such as macular oedema and retinal detachment which might affect the course of hyperopia, were not found in any angiograms of the eyes. Progression of diabetic retinopathy was observed in three patients (patients 2, 5, and 7 ) during the observation period. Three patients developed bilateral background retinopathy. Case 13 had developed retinopathy at the first visit to our outpatient clinic and had required laser photocoagulation at that time. No patient developed proliferative retinopathy during the study.

Table 3 Refractive components

\begin{tabular}{llll}
\hline & $\begin{array}{l}\text { At peak of } \\
\text { hyperopic change }\end{array}$ & Pretreatment value & p Value \\
\hline Corneal anterior curvature (mm) & $7.68(0.22)$ & $7.66(0.20)$ & NS \\
Lens thickness (mm) & $4.27(0.62)$ & $4.31(0.56)$ & NS \\
Anterior chamber depth (mm) & $3.32(0.31)$ & $3.31(0.24)$ & NS \\
Axial length (mm) & $23.54(1.06)$ & $23.47(1.01)$ & NS \\
\hline
\end{tabular}

\section{Discussion}

During intensive hypoglycaemic treatment in the hospital, some diabetic patients complain of disturbance of vision such as difficulty in reading and blurred vision with their own glasses because of refractive changes. If a new prescription for glasses is made at that time, there is a possibility that the new glasses will soon become inadequate. This phenomenon occurs with transient hyperopia due to acute changes in plasma glucose levels.

In diabetic patients, rapid reduction in plasma glucose sometimes aggravates diabetic retinopathy and reduces visual acuity. Although progression of retinopathy occurred in three patients in the present study, there were no abnormal findings such as macular oedema or diabetic maculopathy directly affecting visual acuity, and no patient suffered a decline in corrected visual acuity.

Refractive changes associated with DM are both acute and chronic. Regarding chronic refractive changes in diabetic patients, DukeElder reported that hyperglycaemia led to the development of myopia, while hypoglycaemia led to the development of hyperopia. ${ }^{9}$ However, the available data are conflicting. Many authors who investigated the effect of acute changes in plasma glucose level, have reported that decreasing plasma glucose levels causes hyperopic change. ${ }^{13-6}$ It was also reported that a hyperopic change occurred regardless of whether the plasma glucose level increased or decreased. ${ }^{2}$ Some investigators have observed both myopic and hyperopic changes in diabetic eyes. ${ }^{78}$ Thus, the underlying mechanism of the relation between plasma glucose concentration and refractive change in diabetics remains to be established. Several papers have reported that an abrupt reduction in plasma glucose in diabetic patients with marked hyperglycaemia induced transient hyperopia. ${ }^{12}$ In a study of 10 eyes of five diabetic patients, bilateral transient hyperopia with a maximum refractive change of between 1.1 and 4.9 dioptres, occurred after initiation of strict hypoglycaemic control.

Few studies regarding the effect of rapid correction of hyperglycaemia on refraction in diabetic patients have been conducted. Those which have been published included a small number of subjects, and were conducted as a retrospective study. A study that analyses the refractive error in diabetic patients, and elucidates the underlying mechanism, has not been reported. The present study investigated the clinical course of the refractive change of 28 diabetic eyes during intensive glycaemic control in detail, using many parameters not previously examined. This study revealed that during treatment of hyperglycaemia in diabetic patients, a transient hyperopic change of 0.5 dioptre or more developed in all eyes.

Although refractive errors may occur in adults who have various ocular disorders, the incidence of transient hyperopia among adults with ocular diseases is low. Hyperopia may result from morphological changes in the cornea, axial length, or lens, the three refractive components of the eye. 
Hyperopia may develop as a result of development of a chalazion which presses the cornea, causing central corneal flattening and reducing the refractive power. ${ }^{10}$ In such cases, elimination of pressure would cure the hyperopia. In the present study, the corneal curvature did not significantly change over the follow up period; therefore, corneal involvement in transient hyperopia appears to be unlikely.

A reduction of the axial length could cause hyperopia. An orbital tumour adjacent the posterior pole of the eyeball may reduce the axial length with pressure and cause hyperopia. ${ }^{11}$ Hyperopia secondary to Vogt-KoyanagiHarada syndrome, central serous chorioretinopathy, or retinal detachment is attributable to relative reduction of the axial length due to neurosensory retinal detachment in the macula.

In the present study, fluorescein fundus angiography was performed when the hyperopic change reached 2 dioptres or more and again after refraction was stabilised. The angiographic examinations did not reveal any abnormalities in the macula. In addition, ultrasonographic biometry did not showed any significant changes of the axial length of the eyes. These findings could exclude the possibility that a change in the axial length might be involved in the development of transient hyperopic change.

Lens abnormalities have been suggested as a cause of hyperopia in diabetic patients. In patients with DM, excess glucose in the crystalline lens is converted to sorbitol through the action of aldose reductase. Sorbitol, a sugar alcohol, has poor permeability through membranes and tends to accumulate in the lens. ${ }^{12}{ }^{13}$ When the body rapidly changes from a hyperglycaemic to a hypoglycaemic state, excess sugars which had accumulated in the lens will flow out into the aqueous humour. However, although glucose freely enters the intracellular space, sorbitol, which is less permeable and harder to metabolise, will remain in the lens longer. The difference in osmotic pressure results in the influx of water from the aqueous humour into the lens, causing lenticular swelling with hyperopic refractive changes. This is a possible hypothesis for explaining the occurrence of transient hyperopic changes. Saito et $a l^{1}$ reported that a rapid reduction in blood glucose resulted in transient hyperopia in 10 eyes of five patients with severe diabetes, and that the lens thickness increased significantly and the anterior chamber depth decreased significantly during transient hyperopia. Their findings support this hypothesis. However, the hypothesis which incorporates both morphological and functional changes of the lens is debatable, because an increase in lens thickness would promote myopic changes by reducing lens curvature. The refractive power of a lens is determined by its thickness, anterior and posterior surface curvature, refractive index, and the refractive index of the aqueous humour and vitreous body directly in contact with the lens. Thus, the hypothesis cannot be verified without knowing the effects of each of these contributing factors.

In the present study, the lens thickness did not increase significantly and the anterior chamber depth did not decrease significantly during the occurrence of hyperopic changes. No significant changes were observed in the other optical components-anterior corneal curvature and axial length.

Thus, the transient hyperopic changes in diabetic patients during intensive glycaemic control is attributed not to morphological changes in the cornea, axial length, or lens, but to some intraocular change in refraction. Hyperopia can be caused by a functional change, which may occur in the absence of morphological changes in the lens. The refractive index of the lens changes with ageing and cataract. ${ }^{14}{ }^{15}$ An acute increase or reduction in blood glucose in diabetic patients can sometimes lead to the development of transient cataracts. ${ }^{16-19}$ Thus, a rapid reduction in blood glucose may alter the composition of the lens. An experimental study demonstrated that a decrease in refractive index from 1.42 to 1.40 produced a hyperopic change of 3.2 dioptres; therefore, a slight change in the refractive index produces a significant hyperopic change. ${ }^{20}$ Gwinup and Villarreal $^{4}$ gave an intravenous injection of a $50 \%$ glucose solution $(50 \mathrm{ml})$ to diabetic patients and measured the change in refraction over 90 minutes, and reported that the vision of diabetic patients with phakia became more myopic or less hyperopic, whereas aphakic eyes showed a slight hyperopic change. The definite difference in the eyes with or without a lens indicates that the change in refraction associated with a change in blood glucose level is primarily related to the lens.

However, there is a possibility that changes of intraocular refractive components, including the lens, may cause hyperopia. The eye has two blood-ocular barriers serving to protect against environmental changes: the blood-aqueous barrier, which consists of the iris, vascular endothelial cells, and the ciliary nonpigmented epithelial cells, and the bloodretinal barrier, which consists of the retinal pigment epithelium and the retinal vascular endothelium. If there is an abrupt plasma glucose reduction, a transient difference in osmotic pressure will develop inside and outside of the eye because of the close proximity of the blood-ocular barriers to the eye, and may alter the composition of the lens, aqueous humour and vitreous body. Changes in these components may cause a reduction in the refractive index, leading to hyperopic change.

In the present study, patients who had a higher plasma glucose concentration and higher $\mathrm{HbA}_{1 \mathrm{c}}$ on admission, had a larger maximum hyperopic change. There was a definite positive correlation between the maximum hyperopic change and the daily rate of plasma glucose reduction over the first 7 days after the day of admission. However, there was no significant correlation among the maximum hyperopic change of an eye and each of sex, age, duration of disease, and type of hypoglycaemic therapy. These results indicate that the 
degree of hyperopia is highly dependent on the magnitude of the change in plasma glucose concentration.

Our study revealed that there was a significant correlation between the maximum hyperopic change in an eye and the daily rate of plasma glucose reduction over the first 7 days of treatment, but not with the daily rate of plasma glucose reduction over the first 3 days. This time lag could be attributed to the presence of the blood-ocular barriers. An explanation for the finding that more myopic eyes at baseline had a larger maximum hyperopic change is that myopic eyes have a larger volume with elongated axial length and dysfunctional blood-ocular barrier, and the changes in the composition of the intraocular fluid and the differences in osmotic pressure would be greater in myopic eyes than in eyes having hyperopia as a baseline refractive error. To prove this hypothesis, however, it would be necessary to investigate exactly the change of each optical component such as cornea, anterior chamber depth, lens, and vitreous. Since each of these intraocular tissues is in contact with various adjacent tissues affecting ocular refraction, accurate biometric measurements would be difficult. A study using an animal model may provide useful information.

After beginning intensive glycaemic control for hyperglycaemia in a hospital, diabetic patients frequently have blurred vision and request a prescription of new glasses. However, glasses prepared during the occurrence of transient hyperopic change will not fit after 1 or 2 months. This might create distrust of medical care. The prescription of spectacles should be delayed until a stable refraction is obtained, if possible. If glasses must be prescribed while the refractive status is still unstable, the patient should be informed that the refraction may change over time and that further modifications in the prescription may be needed.

1 Saito Y, Ohmi G, Kinoshita S, et al. Transient hyperopia with lens swelling at initial therapy in diabetes. $\mathrm{Br} F$ Ophthalmol 1993;77:145-8.

2 Eva PR, Pascoe PT, Vaughan DG. Refractive change in hyperglycaemia: hyperopia, not myopia. $\mathrm{Br} \mathcal{F}$ Ophthalmol 982;66:500-5.

3 Willi MJ. Hyperopia and hyperglycemia. Surv Ophthalmol 1996;41:187.

4 Gwinup G, Villarreal A. Relationship of serum glucose concentration to changes in refraction. Diabetes 1976;25:29

5 Keller JT. Hyperopia in diabetes. Arch Ophthalmol 1973;90: 511-12.

6 Marmor MF.Transient accommodative paralysis and hyperopia in diabetes. Arch Ophthalmol 1973;89:419-21.

7 Fledelius HC. Refractive change in diabetes mellitus around onset or when poorly controlled. A clinical study. Acta Ophthalmol (Copenh) 1987;65:53-7.

8 Hugget A. The appearance of the crystalline lens during different stages of transitory changes of refraction. Acta Ophthalmol (Copenh) 1954;32:375-89.

9 Duke-Elder S. Changes in refraction in diabetes mellitus. $\mathrm{Br}$ f Ophthalmol 1925;9:167-87.

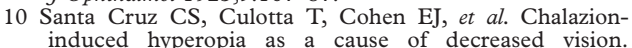
induced hyperopia as a cause of
Ophthalmic Surg Lasers 1997;28:683-4.

11 Friberg TR, Grove AS. Choroidal folds and refractive errors associated with orbital tumors. An analysis. Arch Ophthalmol 1983;101:598-603.

2 Gabbay KH. The sorbitol pathway and the complications of diabetes. N Engl F Med 1973;288:831-6.

13 Kinoshita JH, Merola LO. Osmotic changes caused by the accumulation of dulcitol in the lenses of rats fed with galactose. Nature 1962;194:1085.

14 Hemenger RP, Garner LF, Ooi CS. Change with age of the refractive index gradient of the human ocular lens. Invest Ophthalmol Vis Sci 1995;36:703-7.

15 Smith G, Atchison DA, Pierscionek BK. Modeling the power of the aging human eye. $f$ Opt Soc Am [A] 1992;9:2111-17.

16 Butler PA. Reversible cataracts in diabetes mellitus. $7 \mathrm{Am}$ Optom Assoc 1994;65:559-63.

17 Gelvin JB, Thonn VA. The formation and reversal of acute cataracts in diabetes mellitus. F Am Optom Assoc 1993;64: 471-4.

8 Perkins ES. Cataract: refractive error, diabetes, and morphology. Br f Ophthalmol 1984;68:293-7.

19 Dickey JB, Daily MJ. Transient posterior subcapsular lens opacities in diabetes mellitus. Am $\mathcal{F}$ Ophthalmol 1993;115: $234-8$.

20 Planten J. Changes of refraction in the adult eye due to changing refractive indices of the layers of the lens. Ophthalmologica 1981;183:86-90. 